\title{
Invited commentary on HERN-D-11-00301R3
}

\author{
A. C. de Beaux
}

Received: 20 April 2012 / Accepted: 1 May 2012 / Published online: 16 May 2012

(C) Springer-Verlag 2012

Hernia surgery is going through the 'Classification Revolution'. There is a clear need to be able to compare like with like, whether it be a hernia type, operation technique or a mesh material. Classification can assist with this, and the authors of this study and other before them are to be congratulated on the amount of thought and effort devoted to trying to produce a simple working classification of mesh. The argument put forward for porosity, or more correctly, effective porosity, is a good one, but confidence in the measurement of this parameter and that this parameter only really applies to 2 of the 6 main subgroups within the classification is of concern.

All the products fall under the broad term of 'mesh', but like the collective term 'fruit', comparing apples and oranges is a minefield. The authors would suggest that Type 1 mesh, 'large pores', is the preferred choice for flat meshes. Yet, a mesh that on paper should be in the Type 1 category (Surgipro) following explantation analysis has been reclassified as a Type 2 mesh. This creates problems for new meshes coming to market-they may be Type 1 on paper, but who is responsible for showing that in vivo they behave as Type 1 meshes should? Also, can all industrial mesh companies provide these data, and more importantly, can we compare the data that they provide, not knowing how these data on porosity were generated and whether they are reproducible by independent research groups? The authors offer no real answer to this, including minimum requirements of porosity measurement and testing prior to coming to market.

$60 \%$ effective porosity-the authors acknowledge that this was an educated guess. But no data are given in the paper to suggest that this figure is supported by analysis such as receiver operator curves. Why not $65 \%$ or $55 \%$ for example?

I see this paper as a discussion document. It is not the finished gospel of effective porosity as yet-clearly, more research is required to identify the rules for the optimal mesh (perhaps the rules change for different mesh material, hernia type, patient type and so on). I believe that the authors of this paper, and indeed Hernia, would welcome a vigorous debate of this paper.

\footnotetext{
A. C. de Beaux ( $\square)$

Royal Infirmary of Edinburgh, Edinburgh EH16 4SA, UK

e-mail: adebeaux@doctors.org.uk
} 